\title{
Visual discrimination pretraining facilitates subsequent visual cue/toxicosis conditioning in rats
}

\author{
ANDREW J. DALRYMPLE and BENNETT G. GALEF, JR. \\ McMaster University, Hamilton, Ontario L8S 4K1, Canada
}

\begin{abstract}
The present experiments were undertaken to determine whether rats pretrained to use visual cues in selecting food objects would subsequently learn toxicosis-induced aversions to visually novel foods. We found that rats that had been pretrained to use visual features of their food in selecting palatable items for ingestion rapidly acquired aversions to visually novel foods ingested prior to toxicosis onset. Control subjects, lacking such visual discrimination pretraining, failed to acquire an illness-based aversion to visually novel foods.
\end{abstract}

Norway rats will more readily learn aversions to gustatory than to visual cues experienced prior to onset of toxicosis (Garcia \& Koelling, 1966). Although rats can acquire toxicosis-induced aversions to visual stimuli, acquisition of such aversions generally requires either large numbers of training trials or simultaneous presentation of CS and US (for examples, see Best, Best, \& Mickley, 1973; Mitchell, Kirschbaum, \& Perry, 1975; Morrison \& Collyer, 1974). In contrast, robust taste aversions are acquired following one or two training trials even if experience of taste and illness are separated by many minutes or hours.

Testa and Ternes (1977) have hypothesized that the predisposition of rats to acquire toxicosis-induced aversions to gustatory cues may result from subjects' experience throughout life both of correlations between oral stimuli and gastric events and of the independence of exteroceptive stimuli and gastric events (see also Mackintosh, 1973). Others (Garcia \& Ervin, 1968; Rozin \& Kalat, 1971; Seligman, 1970) have suggested that the predisposition exhibited by rats to associate gustatory cues with toxicosis reflects specialized learning mechanisms evolved in response to environmental contingencies acting as selective pressures on rat populations throughout their evolution.

The first of these models of the origin of the predisposition to associate aversive gastric events with taste

This research was supported by grants from the National Science and Engineering Research Council of Canada and the McMaster University Research Board to B. G. Galef, Jr. We thank Paul Henne, Lynda Ashbourne, and Steven Wigmore for their assistance in filling by hand the more than 10,000 gelatin capsules used in the present experiments and the Parke-Davis Company, Ltd., Brockville, Ontario, for supplying said capsules without charge. The experiments reported here formed part of a dissertation submitted by the first author to McMaster University in partial fulfillment of the requirements of the $\mathrm{PhD}$ degree. Requests for reprints should be sent to Bennett G. Galef, Jr., Department of Psychology, McMaster University, Hamilton, Ontario L8S 4K1, Canada. cues implies that the tendency to form toxicosis-induced aversions to gustatory cues should be readily modifiable by experience; the second model suggests, but does not require, that preexperimental experiences may not have important effects on stimulus selectivity in aversion learning experiments.

The experiments reported below were undertaken to determine whether the probability of rats learning toxicosis-induced aversions to visual cues could be facilitated by prior experience in using visual cues to select ingesta. The outcome indicated that the probability of rats' forming visual cue/toxicosis associations is modifiable by prior experience of visual cues as reliable predictors of the palatability of foods.

\section{EXPERIMENT 1}

In the present experiment, subjects were pretrained, using a simultaneous discrimination procedure, to utilize the visual properties of food objects to select palatable rather than unpalatable items for ingestion. Once subjects were successfully selecting palatable items on the basis of their appearance, they were offered visually novel, familiar-tasting, palatable food objects and then poisoned. If visual discrimination pretraining were found effective in facilitating aversion learning to the visual properties of foods, the existence of an important degree of plasticity in the predisposition of rats to fail to form toxicosis-based aversions to visual cues would be demonstrated.

\section{Method}

Subjects. Subjects were $36150-$ to $200-\mathrm{g}$ male Long-Evans rats acquired from the Canadian Breeding Farms (St. Constant, Quebec).

Apparatus. The experiment was conducted in a two-chambered transparent Plexiglas enclosure illustrated in overhead schematic in Figure 1. The apparatus consisted of a startbox $(25 \mathrm{x}$ $18 \times 13 \mathrm{~cm})$ opening onto a goalbox $(25 \times 25 \times 15 \mathrm{~cm})$ via a guillotine door. Each of two openings $(6 \times 5 \mathrm{~cm})$ in the wall of 


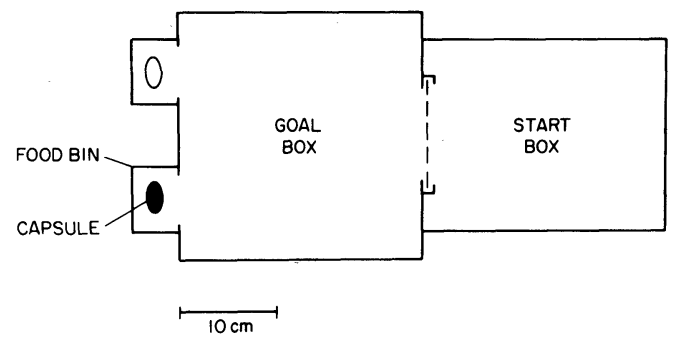

Figure 1. Overhead schematic of the apparatus. Shown with two capsules present in the food bins as during a trial in the discrimination pretraining phase of the experiments.

the goalbox opposite to the startbox door provided access to a food bin $(6 \times 5 \times 5 \mathrm{~cm})$.

Food (powdered Purina Laboratory Chow, packed into No. 2 gelatin capsules of varying color, $.35 \mathrm{~g} /$ capsule) was presented to subjects on the floor of each food bin.

Procedure. Maintenance. For the 2 weeks of habituation, discrimination pretraining, training, and testing, each subject was housed individually, handled daily, and maintained with ad-lib access to water on a restricted feeding schedule (sufficient Purina Laboratory Chow to establish and maintain body weight at approximately $80 \%$ of the body weight of rats of the same strain, age, and sex maintained with ad-lib access to food).

Habituation. For the first 7 days of the habituation phase of the experiment, all subjects were familiarized with the test room and test apparatus during daily 100-min sessions. On each day, subjects were placed in groups of 10 on a table in the experimental room and moved in pairs to spend $20 \mathrm{~min}$ exploring the apparatus.

On Day 8 of habituation, individual subjects were accustomed to eating encapsulated rations by having 10 No. 2 "white opaque" capsules (Parke-Davis, Brockville, Ontario) filled with powdered Purina Laboratory Chow placed in their cages for $1 \mathrm{~h}$. Subjects were further familiarized with experimental procedures on the following day, when each was trained for 10 trials to run from the startbox to retrieve and eat single Purina-Chow-filled "white opaque" capsules from the food bins.

Discrimination pretraining. Each subject was trained for 2 consecutive days ( 20 trials/day) to choose between pairs of visually distinguishable capsules having palatable or unpalatable contents. On each trial, an individual subject was presented two capsules, one light ("white opaque") and one dark ("scarlet opaque"). For half the subjects, the light capsule contained unadulterated powdered Purina Laboratory Chow (palatable contents) and the dark capsule, powdered Purina Laboratory Chow adulterated $4 \%$ by weight with quinine hydrochloride (unpalatable contents). The palatability of the contents of light and dark capsules was reversed for the remainder of the subjects.

A trial was initiated by restraining the subject in the startbox of the apparatus for $30 \mathrm{sec}$. The guillotine door was then opened, the subject was allowed either $300 \mathrm{sec}$ to open a capsule or $60 \mathrm{sec}$ to eat a capsule's contents after opening it. The subject was then returned to the startbox for $30 \mathrm{sec}$.

Each subject was given 20 trials/day, using a noncorrection procedure, until it achieved a criterion of 9 correct choices in 10 consecutive trials. A correct choice was defined as opening and ingesting the contents of the palatable capsule prior to contacting the unpalatable capsule. Any subjects not reaching criterion on Day 2 of discrimination pretraining (two subjects) were discarded.

Training. Twenty-four hours following mastery of the discrimination task, subjects were randomly assigned to one of two studies. Subjects in the experimental group of each study were treated identically, but each study employed a different control procedure.
Subjects $(n=20)$ in both groups in Study 1 were presented, for two trials on each of 2 training days, a single visually novel capsule, half light ("white opaque") and half dark ("scarlet opaque"), containing unadulterated powdered Purina Laboratory Chow. Subjects in the experimental group were poisoned $5 \mathrm{~min}$, and subjects in the control group, $60 \mathrm{~min}$, after ingesting the second visually novel capsule on each training day. Poisoning was accomplished by an intraperitoneal injection of .12-M lithium chloride solution ( $2 \mathrm{ml} / 100 \mathrm{~g}$ body weight).

Subjects $(n=16)$ in both groups in Study 2 were presented, for two trials on each of 2 training days, a single visually novel food object and poisoned $5 \mathrm{~min}$ after ingesting the food object on the second trial. Visually novel food objects presented to subjects in the experimental group were the same (half-light and half-dark) novel capsules used in Study 1 . Visually novel food objects presented to subjects in the control group were made by wetting, kneading, and drying $.35 \mathrm{~g}$ of powdered Purina Laboratory Chow into a capsule-shaped pellet.

Testing. On the test day, we determined the latency of each subject to open a single half-light and half-dark No. 2 gelatin capsule containing unadulterated powdered Purina Laboratory Chow on each of 10 consecutive trials. As during pretraining and training, on each test trial a subject was allowed either $300 \mathrm{sec}$ to open a capsule or $60 \mathrm{sec}$ after it had opened a capsule before it was returend to the startbox for $30 \mathrm{sec}$ prior to initiation of the next trial.

Testing was carried out using a blind procedure insuring that neither the experimenter nor his assistant (who carried subjects from the colony room to the experimental room) knew the group assignment of any subject until the testing of all subjects had been completed.

Data analysis. Because of both within-group bimodality of scores and nonhomogeneity of variance among groups, we used a nonparametric statistic, the Kruskal-Wallace one-way analysis of variance for independent samples (two-tailed), for all data analyses in Experiment 1.

Data presentation. Because of the extreme variability in behavior among subjects within some groups, we found it necessary to transform our data logarithmically to facilitate graphic presentation.

\section{Results and Discussion}

The main results of Experiment 1 are presented in the panels labeled Study 1 and Study 2 in Figure 2. The left-hand panel of Figure 2 shows both group median and individual latencies of control and experimental subjects in Study 1 to open 10 capsules on the test day. As is clear from examination of the figure and as a statistical test confirmed (Mann-Whitney $U=13.0, p<.05$ ), visual discrimination-pretrained subjects poisoned $5 \mathrm{~min}$ following ingestion of a visually novel food exhibited



Figure 2. Individual and group median latencies of subjects in Experiments 1 and 2 to open all 10 capsules during the testing phase of the experiments. 
greater aversions to the visual properties of that food during testing than did those subjects poisoned $60 \mathrm{~min}$ following ingestion of the visually novel food. The fact that the temporal relationship between CS and US during the training phase of the experiment determined whether aversion learning occurred indicates that avoidance of the CS in the testing phase of the experiment depends on a learned association between CS and US, rather than on unconditioned responses to toxicosis in discrimination-pretrained subjects.

The middle panel of Figure 2 presents both group median and individual latencies of subjects in Study 2 to open 10 test capsules during testing. As is clear from examination of the figure, and as a statistical test confirmed (Mann-Whitney $\mathrm{U}=15.0, \mathrm{p}<.02$ ), discriminationpretrained subjects poisoned $5 \mathrm{~min}$ following ingestion of a visually novel food exhibited aversion to the visual properties of that food, but not other foods, during testing. The finding that avoidance of food objects in the testing phase of the experiment is specific to those food objects associated with toxicosis in the training phase of the experiment again indicates that experimental subjects learned to associate the visual properties of their training capsules with toxicosis.

Informal observation of the behavior of subjects also suggested that those in experimental groups in both Studies 1 and 2 formed aversions to the visual properties of visually novel capsules, analogous to the aversions formed to flavor cues associated with toxicosis in standard aversion learning situations. During testing, 15 of 19 subjects in the two experimental groups directed behaviors normally associated with ingestion of unpalatable substances toward the test capsule (e.g., rubbing chin on the floor of the cage, ejection of food from the mouth, excessive salivation, pushing and pawing the capsule, etc.; Grill \& Norgren, 1978). Only 3 of 15 subjects in the two control groups did so. Given that the experimenter made these behavioral observations while ignorant of the group assignment of subjects, the data provide further evidence that subjects in the two experimental groups had formed aversions to the visual properties of ingesta previously associated with poison.

\section{EXPERIMENT 2}

While data from Experiment 1 provide evidence that the avoidance of test capsules exhibited by experimental subjects during testing was the result of association learning during the training phase of the experiment, these data provide no evidence of the necessity of discrimination pretraining for subsequent aversion learning during training. In the present experiment, we yoked the pretraining experience of each subject to that of a subject in one of the experimental groups of Experiment 1 , so that each yoked subject received quinineadulterated capsules on the same discrimination pretraining trials as the experimental subject to which it was yoked. Bitter capsules ingested by the yoked-control subjects were not, however, visually distinctive, so yoked-control subjects were not explicitly trained to use visual cues in selecting items for ingestion. If visual discrimination pretraining is important in facilitating subsequent visual cue avoidance learning, then subjects in the present study, which were not pretrained to employ visual properties of their food objects in diet selection, should fail during testing to exhibit aversions to visual cues experienced prior to toxicosis during training.

\section{Method}

Subjects. Subjects were eight male Long-Evans rats acquired from the Canadian Breeding Farms at 150-200 g.

Procedure. Subjects in the present experiment were treated identically to those in the experimental group of Study 2 of Experiment 1, except during the discrimination pretraining phase of the experiment. Following habituation, each subject in the present experiment was yoked to one of the eight experimental subjects in the designated model group. Each yokedcontrol subject received quinine-adulterated capsules on the same pretraining trials on which its pairmate had selected quinineadulterated capsules for ingestion. Yoked subjects, however, were prevented from learning to use visual cues to distinguish palatable from unpalatable ingesta. While each yoked-control subject received the same number of "scarlet opaque" and "white opaque" capsules as its model from the experimental group of Study 2 of Experiment 1, the color of capsules presented to yoked subjects did not correlate with the palatability of capsule contents; equal proportions of palatable and unpalatable capsules presented to yoked subjects were scarlet or white in color.

Following discrimination pretraining, all yoked subjects were trained and tested using the same procedures employed with subjects in Experiment 1.

\section{Results and Discussion}

The main results of Experiment 2 are presented in Figure 2, in the histogram labeled "yoked." The right-hand panel of Figure 2 shows both median and individual latencies of subjects in Experiment 2 to open 10 unadulterated capsules on the day of testing. As is clear from examination of the figure, yoked-control subjects exhibited markedly shorter latencies to ingest capsules during testing than did subjects in their model group, the experimental group of Experiment 1, Study 2 (Wilcoxon matched-pairs signed-rank test, $T=1$, $\mathrm{p}<.02)$.

The results indicate that discrimination pretraining, rather than exposure to bitter taste or scarlet capsules during pretraining trials, was responsible for the visual cue/toxicosis association learning exhibited by experimental subjects in Experiment 1.

\section{GENERAL DISCUSSION}

The results of the experiments described above indicate that visual discrimination pretraining increases the subsequent probability of rats learning aversions to visually novel food objects ingested prior to toxicosis onset. The data do not, however, provide sufficient information to permit determination of the process or processes underlying this enhancement of visual cue/ toxicosis aversion learning.

Galef and Osborne (1978) have suggested that the usual 
failure of rats to form aversions to the visual properties of foods experienced prior to toxicosis may result, at least in part, from a failure of rats to attend to the visual properties of food objects. The findings reported above are consistent with such an interpretation. If, as seems likely, requiring rats to employ visual cues in selecting palatable food items increases the attention that rats direct to the visual properties of their food, then one would expect, on the Galef and Osborne hypothesis, facilitation of visual cue/toxicosis conditioning by visual discrimination pretraining.

Alternatively, it is possible: (1) that aversions learned to light or dark bitter-tasting capsules, experienced during the discrimination pretraining phase of the experiment, generalized to the half-light, half-dark training capsules and (2) that this bittertaste-induced, generalized aversion facilitated subsequent toxicosis-based aversion learning during the training phase of the experiment.

Regardless of the process proposed to account for the observed effect of visual discrimination pretraining on subsequent visual cue aversion learning, the results of the present experiment clearly indicate that the probability of acquiring a toxicosisinduced aversion to visual cues of rats is modifiable by their previous experience of visual cues as predictors of palatability of food objects.

\section{REFERENCES}

Best, P. J., Best, M. R., \& Mickley, G. A. Conditioned aversion to distinct environmental stimuli resulting from gastrointestinal distress. Journal of Comparative and Physiological Psychology, 1973, 82, 250-257.

Galef, B. G., JR., \& Osborne, B. Novel taste facilitation of the association of visual cues with toxicosis in rats. Journal of Comparative and Physiological Psychology, 1978, 92, 907-916.

GARCIA, J., \& ERvin, F. R. Gustatory-visceral and telereceptorcutaneous conditioning: Adaptation in internal and external milieus. Communications in Behavioral Biology, 1968, 1, 389-415.

Garcia, J., \& Koelling, R. A. Relation of cue to consequence in avoidance learning. Psychonomic Science, 1966, 4, 123-124.

Grill, H. J., \& Norgren, R. The taste reactivity test. I. Mimetic responses to gustatory stimuli in neurologically normal rats. Brain Research, 1978, 143, 263-279.

MaCkintosh, N. J. Stimulus selection: Learning to ignore stimuli that predict no change in reinforcement. In $\mathbf{R}$. A. Hinde \& J. Stevenson-Hinde (Eds.), Constraints on learning. New York: Academic Press, 1973.

Mitchell, D., Kirschbaum, E. H., \& Perry, R. L. Effects of neophobia and habituation on the poison-induced avoidance of exteroceptive stimuli in the rat. Journal of Experimental Psychology: Animal Behavior Processes, 1975, 104, 47-55.

Morrison, G. R., \& Collyer, R. Taste-mediated conditioned aversion to an exteroceptive stimulus followin $\mathrm{LiCl}$ poisoning. Journal of Comparative and Physiological Psychology, 1974, 86, 51-55.

Rozin, P., \& Kalat, J. W. Specific hungers and poison avoidance as adaptive specializations of learning. Psychological Review, 1971, 78, 459-486.

Seligman, M. E. P. On the generality of the laws of learning. Psychological Review, 1970, 77, 406-418.

Testa, T., \& Ternes, J. W. Specificity of conditioning mechanisms in the modification of food preferences. In L. M. Barker, M. R. Best, \& M. Domjan (Eds.), Learning mechanisms in food selection. Waco, Tex: Baylor University Press, 1977.

(Received for publication August 26, 1981.) 